\title{
Book Review: Kurath, Monika; Bürgin, Reto (Hrsg.) (2019): Planung ist unsichtbar. Stadtplanung zwischen relationaler Designtheorie und Akteur-Netzwerk-Theorie. Bielefeld: transcript Verlag. 250 Seiten
}

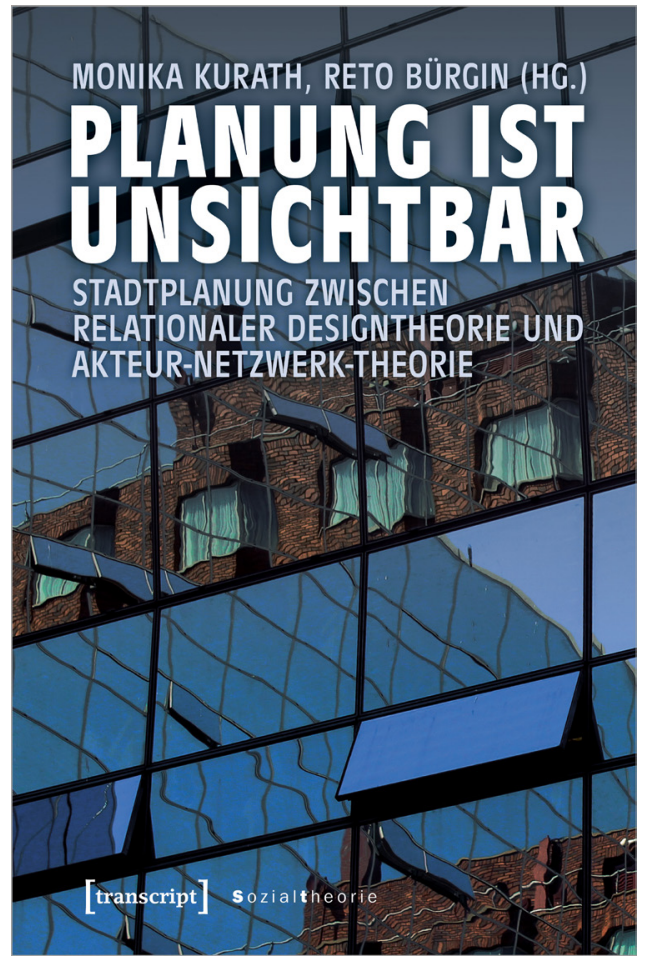

Die Planungswissenschaften nehmen im Reigen der akademischen Disziplinen eine Sonderstellung ein. Denn wie in kaum einer anderen Disziplin sind ihre Akteure Planerinnen und Planer - ihr eigener Untersuchungsgegenstand und erforschen in erster Linie sich und ihr eigenes Tun. Um auf diese ,berufsnahe' Forschung einen möglichst reflektierten Blick werfen zu können, setzt die Planungstheorie spätestens seit dem communicative turn auf theoretische Impulse aus benachbarten Disziplinen, um so gängige Interpretationen des eigenen Planungshandelns zu hinterfragen und Planung aus alternativen Perspektiven neu zu verstehen.

Der vorliegende Sammelband „Planung ist unsichtbar", herausgegeben von Monika Kurath und Reto Bürgin, widmet sich solch einem Theorieimport in die Planungswissenschaften: der Akteur-Netzwerk-Theorie (ANT). In der Einleitung verorten sie diese als Teil eines relationalen Planungsverständnisses, das durch eine „sozio-materielle Denkweise neue Perspektiven auf die Planung" (S. 24) bietet. Analog zu dem material turn in den Sozialwissenschaften wird demnach auch in der Planung der Fokus vermehrt auf die Rolle materialisierter und verkörperter Interaktionen gelegt und deren Bedeutung für planerische Prozesse genauer betrachtet.

Der Sammelband adressiert diesen Theorieimport aber nicht ausschließlich als epistemologische Innovation. Vielmehr plädieren die Herausgeberin und der Herausgeber dafür, die Debatten um die Akteur-Netzwerk-Theorie und relationale Planung auch als Wiederbelebung früherer Diskurse zu verstehen, und rücken dabei explizit die Designtheorie des Schweizer Soziologen Lucius Burckhardt ins Scheinwerferlicht. Damit hebt sich der Sammelband von dem häufig im deutsch-

*Corresponding author: Dr. Ulrike Mackrodt, Niederkirchnerstraße 5, 10117 Berlin, Deutschland, E-mail: ulrike.mackrodt@alumni.hu-berlin.de 
sprachigen Diskurs gewählten Ansatz ab, theoretische Debatten aus dem angelsächsischen Raum zu (re-) importieren. Stattdessen zeigt er wichtige Parallelen zwischen zeitlich (und sprachlich) getrennten Diskurssträngen auf, die sonst nur allzu oft verborgen bleiben.

Um diese Parallelen sichtbar zu machen, widmet sich Reto Bürgin im ersten Beitrag des Bandes dem Werk Burckhardts und weist inn als Vordenker relationaler Planung aus. Insbesondere dessen bekannter Aufsatz „Design ist unsichtbar" aus dem Jahr 1980 hat für Bürgin die Grundlagen für eine Designtheorie gelegt, die nicht nur das Ergebnis, sondern den Prozess der Gestaltung in den Fokus rückt. Burckhardt forderte, „die sozio-materiellen Interaktionen und Auswirkungen von Design zu berücksichtigen" (S. 42). Nicht zuletzt der Titel des Sammelbandes "Planung ist unsichtbar" leitet sich unverkennbar von diesem Aufsatztitel Burckhardts ab.

So charmant dieses Sprachspiel auch ist, so stiftet es dennoch ein wenig Verwirrung bei der Lektüre. Denn während Burckhardt seinerzeit auf die „unsichtbare [...] institutionell-organisatorische Komponente" (S. 47) des Gestaltens jenseits des Materiellen aufmerksam machen wollte, fordert der heutige Diskurs um den material turn ja gerade die Rückbesinnung auf die Dinglichkeit und Körperlichkeit allen gesellschaftlichen Tuns. Planung aus dem Blickwinkel des material turn darf gerade nicht unsichtbar sein (bzw. bleiben), sondern muss sichtbar gemacht werden. Die Adaptation des Burckhardt-Titels für den Titel des Sammelbands erscheint der Rezensentin daher weniger glücklich.

Davon unbenommen präsentiert sich der Sammelband mit seiner Verbindung beider Diskursstränge um Akteur-Netzwerk-Theorie und Burckhardts Designtheorie als eine inspirierende Lektüre. So konstatiert Michael Guggenheim in seinem Nachwort richtigerweise, dass „Lucius Burckhardt [...] ein relationaler Planungstheoretiker avant la lettre" (S. 239) war und ordnet damit die neueren ANT-inspirierten Forschungen in der Planung in einen bereits länger währenden Diskurs um das Zusammenspiel materieller und institutioneller Komponenten ein. Theoretische Weiterentwicklungen präsentieren sich vor diesem Hintergrund nicht zwingend als Neuerfindungen des Rades, sondern eben auch als Rückbesinnungen, was ihren Mehrwert in keiner Weise schmälert. Vielmehr werden so die Anschlussfähigkeit wissenschaftlicher Wissensproduktion erhöht und historische Ähnlichkeiten sichtbar gemacht.

Dieses ,Sichtbarmachen' zieht sich als roter Faden durch die weiteren sechs thematischen Beiträge des Sammelbandes. Jonas Müller beleuchtet mit seiner ethnographisch angelegten Forschung die Hintergrundar- beit, „die Planung in Verwaltungen und Planungsbüros leistet" (S. 73), und macht sie damit sichtbar. Indem er bewusst nicht die öffentlichen Formate der Partizipation empirisch untersucht, sondern die informellen Praktiken der Verwaltung, zeigt er (un)ordentliche Prozesse auf, in denen Wissen produziert und Planung vollzogen wird. Nikolai Roskamm gibt in seinem theoretisch ausgerichteten Aufsatz einen argumentativ dichten Überblick über die „Zuwendung zum Ding und zum Objekt“ verschiedener Theoretiker, unter anderen von Emile Durkheim, Martin Heidegger und Jacques Lacan. Insbesondere die Ausführungen zu Bruno Latour - als prägendem Denker der Akteur-Netzwerk-Theorie - geben wertvolle Referenzpunkte für die Lektüre der weiteren empirischen Beiträge, so zum Beispiel das Konzept der Politisierung von Dingen (S. 101). Anna-Lisa Müller widmet sich in ihrem empirischen Beitrag der Sichtbarkeit und Materialität von street art im städtischen Raum und ihrer Bedeutung in Planungsprozessen. Mithilfe des theoretischen Rahmens der Science and Technology Studies nähert sie sich dabei dem „Verhältnis von Sozialität und Materialität" (S. 111) von street art und plädiert in einer weiten Lesart von Planung dafür, Künstlerinnen und Künstler von street art als Teil des Netzwerks „Stadtentwicklung“ zu verstehen.

Christian Peer gibt empirische Einblicke in einen planerischen Leitbildprozess in Wien, der mehrfache Änderungen und Anpassungen durchlaufen hat. Er konstatiert treffend, dass solche dynamischen Planungsprozesse mit der Akteur-Netzwerk-Theorie „als eine Art Tanz imaginiert" (S. 139) werden können, bei dem „stabile und weniger stabile Zustände im Verlauf eines Planungsprozesses" fassbar werden (S. 139). Dies verdeutlicht, dass eine ANT-basierte Analyse eher selten modellhafte oder übertragbare Pfadbeschreibungen für zukünftige Planungsprozesse liefert. Stattdessen wird mit der AkteurNetzwerk-Theorie die Komplexität von Planungsprozessen besser fassbar, was wiederum „eine besondere Art der Sichtbarkeit" (S. 152) ermöglicht.

Die Sichtbarkeit von Planungsprozessen spielt auch in einem zweiten empirischen Beitrag aus Wien eine zentrale Rolle. Monika Kurath und Julio Paulus erörtern anhand zweier Beteiligungsprozesse zur Umgestaltung öffentlicher Räume, „wie Vorstellungen einer lebenswerten Stadt" (S. 186) in Vor-Ort-Beteiligungsformaten sichtbar gemacht und damit Gegenstand planungspolitischer Aushandlung werden können. Dabei argumentieren sie keineswegs rein affirmativ für die Nutzung solcher Formate, sondern zeigen auch die Grenzen eines materiellen Partizipationsansatzes auf, der nicht zwangsläufig positive Auswirkungen auf das Planungsergebnis haben muss. 
Hanna Katharina Göbe/ wählt einen ähnlichen argumentativen Zugang zu ihrem Hamburger Fallbeispiel und untersucht, welche Rolle die Körperlichkeit von Planungsbeteiligten in einer partizipativen künstlerischen Intervention in St. Pauli spielt. Dabei stellt sie treffend dar, dass diese materialisierte Beteiligungs-Intervention die Aufmerksamkeit auf die Körperlichkeit aller beteiligten Akteure gelenkt hat. Die Planungssphäre, die sonst „als das rationalisierte Feld der Entkörperlichung und Entsinnlichung dargestellt" wird (S. 219), wird somit über den Umweg der künstlerischen Beteiligung selbst sichtbar gemacht.

Auch der Beitrag von Jens Lachmann verdeutlicht, wie sichtbare Artefakte Einfluss auf Planungsprozesse nehmen können. Er skizziert die Karriere der Stadtökologie als Wissenschaftsdisziplin „im abgeschlossenen Universum" (S. 178) West-Berlins und zeigt eindrücklich auf, wie diese zu einem wichtigen Akteur im Agenda-Setting der Stadtplanung wurde. Indem das Institut für Stadtökologie der Technischen Universität Berlin auf das Untersuchungsgebiet West-Berlins beschränkt war, fanden umfangreiche Biotopkartierungen beinahe ausschließlich innerhalb des Stadtgebiets statt. Diese Vielzahl städtischer Biotopkartierungen übersetzte sich im politischen Raum in ein Berliner Artenschutzprogramm, womit dem planerischen Instrument des Flächennutzungsplanes eine konkurrierende Sichtbarkeit in Bezug auf mögliche Entwicklungsflächen gegenübergestellt wurde.

Der Sammelband präsentiert sich insgesamt als ein empirisch facettenreiches Plädoyer für das Sichtbarmachen von Planungsprozessen in ihrer Materialität und Körperlichkeit. Die Beiträge belegen eindrücklich, wie vielfältig und auch kontrovers diese Einflüsse sein können. Während der Sammelband seinen Schwerpunkt nicht auf eine differenzierte Theoriedebatte legt, zeigt er auf, dass sich die Akteur-Netzwerk-Theorie „als hochgradig anschlussfähiges, bewegliches Theoriegebäude nutzen" lässt (S. 150). Damit wird es vor allem möglich, die Flexibilität, Wandelbarkeit und Kontingenz von Planungsprozessen zu untersuchen und die Basis für ein besseres Verständnis politischer Aushandlungsprozesse um die Gestaltung städtischer Räume zu schaffen. 